\title{
Single dose
}

\section{pharmacokinetics of the} \section{transdermal rotigotine patch} in patients with impaired renal function

\section{Correspondence}

Dr Willi Cawello, Global Biostatistics, UCB Biosciences $\mathrm{GmbH}$, Alfred-Nobel-Str. 10, 40789 Monheim, Germany.

Tel.: +49 2173481480

Fax:+49 2173481283

E-mail:willi.cawello@ucb.com

Keywords

dopamine agonist, pharmacokinetics, renal impairment, rotigotine, transdermal delivery

\section{Received}

8 September 2010

Accepted

10 June 2011

Accepted Article

27 June 2011

${ }^{1}$ UCB Biosciences, Monheim, Germany, ${ }^{2}$ Department of Nephrology, Jagiellonian University, Krakow and ${ }^{3}$ monipol sp.z.o.o., Center for Clinical Pharmacology Research, Krakow, Poland

\section{WHAT IS ALREADY KNOWN ABOUT THIS SUBJECT \\ - The transdermal dopamine agonist rotigotine has been shown to be an efficacious and well tolerated treatment for Parkinson's disease and restless legs syndrome in clinical trials. \\ - The prevalence of Parkinson's disease and restless legs syndrome increases with age. - Renal function deteriorates with age and can influence drug treatment. \\ - Many patients with end-stage renal disease experience restless legs syndrome.}

\section{WHAT THIS STUDY ADDS}

- The pharmacokinetic profiles of the active substance, unconjugated rotigotine, were similar in healthy subjects and subjects with renal impairment.

- The results suggest that no dose adjustments are required for transdermal rotigotine in subjects with different stages of chronic renal insufficiency including patients on haemodialysis.

\section{AIM}

To evaluate the influence of different stages of chronic renal insufficiency on the pharmacokinetics and safety/tolerability of the transdermally applied dopamine agonist rotigotine in an open label group comparison including 32 subjects (healthy, mild, moderate or severe impairment of renal function and patients with end-stage renal insufficiency requiring haemodialysis).

\section{METHODS}

All subjects received a single transdermal $10 \mathrm{~cm}^{2}$ patch $(24 \mathrm{~h}$ patch-on period) containing $4.5 \mathrm{mg}$ rotigotine (nominal drug release

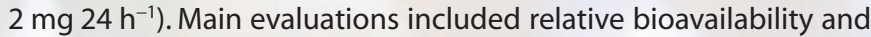
renal elimination of rotigotine and its metabolites.

\section{RESULTS}

Point estimates for the ratios between the groups with moderate to severe renal impairment and healthy subjects for the pharmacokinetic parameters $\operatorname{AUC}\left(0, t_{\text {last }}\right)$ and $C_{\max }$ for the active substance unconjugated rotigotine were near 1:0.88 for AUC and 0.93 for $C_{\max }$ for moderate renal impairment, 1.14 and 1.18 for severe renal impairment and 1.05 and 1.25 for end-stage renal insufficiency requiring haemodialysis. There was no correlation of these parameters with creatinine clearance. The amount of unconjugated rotigotine excreted into urine and renal clearance decreased with increasing severity of renal insufficiency but had no observable effect on total clearance as the amounts excreted were below $1 \%$ of the administered dose. Occurrence of adverse events did not increase with the degree of renal insufficiency.

\section{CONCLUSIONS}

The pharmacokinetic profiles of unconjugated rotigotine were similar in healthy subjects and subjects with impaired renal function indicating that no dose adjustments are required for transdermal rotigotine in patients with different stages of chronic renal insufficiency including patients on haemodialysis. 


\section{Introduction}

The non-ergolinic dopamine agonist rotigotine with activity across $D_{1}$ through $D_{5}$ receptors as well as select adrenergic and serotoninergic sites was developed as a silicone-based matrix-type transdermal system to provide continuous drug delivery with maintenance of stable plasma concentrations over $24 \mathrm{~h}$ [1]. Continuous drug delivery potentially minimizes pulsatile stimulation of striatal dopamine receptors which is implicated in the development of motor fluctuations and dyskinesia [2]. Following patch application, on average approximately half of the administered rotigotine dose $(46 \%)$ is systemically absorbed [3]. Rotigotine is then rapidly metabolized, mainly by conjugation (sulphation and glucuronidation) [4]. A second pathway is the formation of $\mathrm{N}$-desalkyl metabolites with subsequent conjugation [4]. There is no pharmacokinetic interaction with levodopa/carbidopa [5] and rotigotine can be co-administered with the antiemetic domperidone without changes in the pharmacokinetics of rotigotine [6]. Randomized, placebo-controlled studies have demonstrated efficacy in early Parkinson's disease (PD) as monotherapy $[7,8]$ and in combination with levodopa in advanced patients $[9,10]$. Efficacy has also been shown for the treatment of restless legs syndrome (RLS) [11, 12].

Both PD and RLS prevalence increase with age. Overall the prevalence of PD in seven European populationbased studies was $1.8 \%$ in patients over the age of 65 years, ranging from $0.6 \%$ for those aged 65 to 69 years up to $2.6 \%$ for the 85 to 89 year age group [13]. The increase in RLS prevalence between young and older age is two to three fold [14]. RLS prevalence is also high in dialysis patients, and patients suffering from both end-stage renal disease (ESRD) and RLS have an increased risk of mortality [15].

Elderly PD and RLS patients are likely to suffer from other age-related impairments, as most major body systems are affected by advancing age. Changes in renal function and anatomy occur with age [16] which have important implications for drug therapy in the elderly [17]. This phase I study evaluated the influence of different stages of chronic renal insufficiency on the pharmacokinetics and safety/tolerability of the transdermally applied dopamine agonist rotigotine.

\section{Methods}

\section{Study population and design}

This open label, single dose, phase I group comparison study between healthy subjects and subjects with chronic renal insufficiency (not having a diagnosis of PD or RLS) was conducted at two trial sites (Department of Nephrology Jagiellonian University Hospital CM UJ and Centre for Clinical Pharmacology Research monipol, both Krakow,
Poland) between June 2003 and February 2004. Caucasian adults $\geq 18$ and $\leq 75$ years of age with a body mass index (BMI) between 18 and $34 \mathrm{~kg} \mathrm{~m}^{-2}$ who were willing and able to comply with all study requirements were included in the study. Females of childbearing potential could participate in the study provided they were willing to use a double barrier contraceptive method (no oral contraceptives were permitted), were not pregnant, nursing or on hormone replacement therapy. Healthy subjects with no previous or present condition of clinically relevant medical or psychiatric abnormalities and serum chemistry parameters within the normal range were allowed to participate. For exclusion criteria see supplementary online information. The study was conducted in accordance with Good Clinical Practice and the Declaration of Helsinki. The protocol and its amendments were approved by the Bioethics Committees of the Jagiellonian University and the Medical Doctors' Chamber Krakow, Poland. EMEA and FDA guidance on studies in patients with impaired renal function was also followed $[18,19]$. Written informed consent was obtained from all participating subjects.

An eligibility assessment including physical examination, vital signs, 12-lead electrocardiogram (ECG), alcohol and drug screening, pregnancy test, infection serology and safety laboratory parameters was performed within 14 days prior to the treatment period; urine was collected over a $24 \mathrm{~h}$ period within 10 days prior to administration of study medication for the determination of creatinine clearance $\left(\mathrm{CL}_{\mathrm{cr}}\right)$. Eligible subjects were hospitalized for the entire treatment period (day -1 to day 4). Eligibility was confirmed on day -1 (day before first treatment). Eligible subjects were then assigned according to their $\mathrm{CL}_{c r}$ values to one of the following five groups: $A$ ) healthy subjects $\left.\left(\mathrm{CL}_{\mathrm{cr}} \geq 80 \mathrm{ml} \mathrm{min}^{-1}\right), \mathrm{B}\right)$ mild impairment of renal function

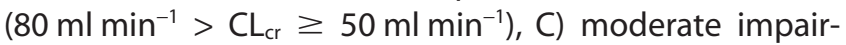
ment of renal function $\left(50 \mathrm{ml} \mathrm{min}^{-1}>\mathrm{CL}_{\mathrm{cr}} \geq 30 \mathrm{ml} \mathrm{min}^{-1}\right)$, D) severe impairment of renal function $\left(\mathrm{CL}_{\mathrm{cr}}<30 \mathrm{ml} \mathrm{min}^{-1}\right.$, no dialysis required) and $\mathrm{E}$ ) end-stage renal insufficiency requiring haemodialysis $\left(\mathrm{CL}_{\mathrm{cr}}<15 \mathrm{ml} \mathrm{min}{ }^{-1}\right)$. Groups were matched by gender and age where possible. The permitted difference in mean age between the groups was \pm 10 years. A follow-up examination was performed 5 to 10 days after application of the study medication.

Treatment consisted of one transdermal $10 \mathrm{~cm}^{2}$ patch

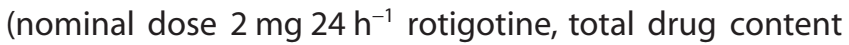
$4.5 \mathrm{mg}$, Schwarz Biosciences, UCB Group, Germany). In the morning of the treatment day, subjects received a single application to the ventral/lateral abdomen with a patch-on period of $24 \mathrm{~h}$. Healthy subjects were not allowed to receive medication other than the study drug during the investigation. Subjects with renal impairment continued their personal medication essential to the treatment of the underlying disease and concomitant diseases. All medication had to be administered for at least five half-lives before study entry to ensure steady-state conditions. Steroids, oestrogens, gestagens, St John's Wort, barbiturates 
and rifampicin had to be withdrawn from at least 4 weeks prior to the study.

Patients in group $\mathrm{E}$ received a $4 \mathrm{~h}$ extracorporeal haemodialysis $22 \mathrm{~h}$ after patch application with a Hemoflow F7 or F8 membrane (Fresenius Medical Care, Germany). The dialysate flow rate was $500 \mathrm{ml} \mathrm{min}$.

\section{Sample collection and analytical methods}

Blood samples for the evaluation of rotigotine and conjugated rotigotine metabolites (rotigotine sulphate, rotigotine glucuronide) were collected before patch application and at 1,2, 4, 8, 12, 16, 23.5, 24 (prior to patch removal), 26, $28,30,36,48$ and $60 \mathrm{~h}$ post application in groups A-D. For the evaluation of $\mathrm{N}$-desalkyl metabolites ( $\mathrm{N}$-despropyl and $\mathrm{N}$-desthienylethyl rotigotine and their conjugates) an additional $5.5 \mathrm{ml}$ of blood were collected at the $23.5 \mathrm{~h}$ time point. Patients in group $\mathrm{E}$ were started on haemodialysis $22 \mathrm{~h}$ after patch application. Blood samples for this group were collected before patch application and 6, 12, 22 (prior to start of dialysis), 24 (prior to patch removal), 26, 28, 30, 36,48 and $60 \mathrm{~h}$ after patch application. At the $22 \mathrm{~h}$ time point additional blood was collected for $\mathrm{N}$-desalkyl metabolite evaluation. For the assessment of extracorporeal extraction of rotigotine and its metabolites, two blood samples were taken at the $26 \mathrm{~h}$ time point (at the end of a $4 \mathrm{~h}$ dialysis), one from the arterial inlet and one from the venous outlet of the dialyser. An aliquot of the dialysis fluid was collected to quantify the total amount of rotigotine (conjugated and unconjugated) extracted by the dialysis. For the assessment of renal excretion of rotigotine and its metabolites urine was collected prior to patch application and for the following intervals post application: 0-4, 4-8, 8-12, 12-24, 24-36, 36-48, and 48-72 h. All samples were analysed by liquid chromatography with tandem mass spectrometry (LC-MS/MS), for details see supplementary information. To obtain conjugated rotigotine concentrations, the difference between total rotigotine and unconjugated rotigotine was calculated.

Quantitative determination of the percentage of protein binding of rotigotine in human plasma was carried out using an equilibrium dialysis procedure (see supplementary information).

Apparent doses (as an estimate for the amount of drug absorbed within $24 \mathrm{~h}$ ) were determined from the used patches as the difference between initial drug content of the patch and the residual drug amount in the used patch after the $24 \mathrm{~h}$ patch-on time.

\section{Outcome parameters}

Primary outcome parameters were the plasma and urine concentrations of unconjugated and conjugated rotigotine and its $\mathrm{N}$-desalkyl metabolites, and the pharmacokinetic parameters peak plasma concentration $\left(C_{\max }\right)$, area under the concentration-time curve from time 0 to the last time point with a quantifiable concentration $\left(A \cup C\left(0, t_{\text {last }}\right)\right)$, terminal half-life $\left(t_{1 / 2}\right)$ and the unbound fraction $\left(f_{u}\right) . C_{\max }$ was obtained by visual inspection of the plasma concentration-time profiles, $\mathrm{AUC}\left(0, t_{\text {last }}\right)$ was determined using the linear trapezoidal rule. The terminal rate constant $\lambda_{z}$ for the calculation of $t_{1 / 2}$ was obtained by log-linear regression and $f_{\mathrm{u}}$ was calculated from the protein binding data.

Secondary parameters were $\operatorname{AUC}\left(0, t_{\text {last }}\right)$ and $C_{\max }$ normalized by bodyweight and apparent dose $\left(A \cup C\left(0, t_{\text {last }}\right)_{\text {norm }}\right.$ and $\left.C_{\text {max, norm }}\right), \operatorname{AUC}\left(0, t_{\text {last }}\right)$ extrapolated to infinity $\operatorname{AUC}(0, \infty)$ was not reported if the terminal area beyond the last quantified sample was greater than $20 \%$ of the total $\operatorname{AUC}(0, \infty))$, time to maximum plasma concentration $\left(t_{\max }\right)$, apparent volume of distribution during the terminal disposition phase $\left(V_{z} / F\right)$, and total clearance of the medication from plasma (CL/F), the total amount excreted in urine (Ae), and renal clearance $\left(\mathrm{CL}_{R}\right)$ of rotigotine and its main metabolites. Furthermore, the extraction of rotigotine by dialysis was determined by measurement of plasma concentrations before and after dialysis and by measurement of concentrations in the dialysis fluid. $\mathrm{CL}_{R}$ was calculated as the quotient of $A$ e to $A \cup C(0, \infty)$, and $C L / F$ by applied dose over $\operatorname{AUC}(0, \infty)$.

Safety and tolerability parameters were repeatedly assessed throughout the study by means of vital signs, 12-lead ECG, physical examination and clinical laboratory parameters. All adverse events (AEs) were recorded and monitored. Details about assessments of skin tolerability and patch adhesiveness can be found in the supplementary online file.

\section{Statistical analysis}

Data analysis was carried out using the SAS program (SAS Institute, Cary, NC, USA; versions 8.2 and 9.1). Owing to the exploratory nature of the present study, no formal sample size calculation was undertaken.

All subjects who completed treatment and showed no major protocol deviations were included in the analysis (per protocol (PP) population). Plasma concentration data below lower limit of quantification (LOQ) were substituted by zero. The pharmacokinetic parameters $\operatorname{AUC}\left(0, t_{\text {last }}\right)$ and $C_{\max }$ of both unconjugated and conjugated rotigotine were logarithmically transformed and subjected to an analysis of variance (ANOVA) with the fixed factor 'group'. Estimated ratio of means (least squares means) and $90 \%$ confidence intervals (Cls) were calculated for the back-transformed data for group comparison. Conjugated and unconjugated $C_{\max }, \operatorname{AUC}\left(0, t_{\text {last }}\right), t_{1 / 2}$ and $f_{\mathrm{u}}$ were plotted against creatinine clearance and the corresponding Spearman's rank correlation coefficients $\left(\rho_{s_{p}}\right)$ were calculated. The unbound fraction $\left(f_{\mathrm{u}}\right)$ was also plotted against serum albumin concentration and evaluated in the same manner. Secondary variables were analysed in a descriptive manner. Parameters $t_{1 / 2}, \mathrm{CL} / F, \mathrm{CL}_{R}$, Ae and $V_{z} / F$ for conjugated and unconjugated rotigotine were also plotted against $\mathrm{CL}_{\mathrm{cr}}$ and the corresponding Spearman's rank correlation coefficients were calculated. Calculation of plasma concentration 


\section{Table 1}

Demographic characteristics of the study population

\begin{tabular}{|c|c|c|c|c|c|}
\hline & Healthy & $\begin{array}{l}\text { Mild impairment } \\
\text { of renal function }\end{array}$ & $\begin{array}{l}\text { Moderate impairment } \\
\text { of renal function }\end{array}$ & $\begin{array}{l}\text { Severe impairment } \\
\text { of renal function }\end{array}$ & $\begin{array}{l}\text { End-stage renal insufficiency } \\
\text { requiring haemodialysis }\end{array}$ \\
\hline Number of patients & 8 & 1 & 7 & 8 & 8 \\
\hline Age (years) & $37.6(17.2)$ & 39 & $55.4(11.2)$ & $41.5(13.5)$ & $49.0(8.7)$ \\
\hline Bodyweight (kg) & $71.0(9.3)$ & 78.0 & $77.7(9.7)$ & $73.8(12.7)$ & $71.0(9.0)$ \\
\hline Height (m) & $1.67(0.09)$ & 1.78 & $1.71(0.11)$ & $1.70(0.03)$ & $1.71(0.06)$ \\
\hline Body mass index $\left(\mathrm{kg} \mathrm{m}^{-2}\right)$ & $25.5(3.7)$ & 24.6 & $26.7(3.8)$ & $25.6(4.2)$ & $24.3(3.4)$ \\
\hline
\end{tabular}

Data are expressed as number of patients or means (SD).

medians and geometric mean and geometric CV of pharmacokinetic parameters were selected to describe the point estimates and variability for each group.

All patients who received the study medication were included in the safety and tolerability assessment. Adverse events were encoded according to the WHO-Adverse Reaction Terms dictionary (WHO-ART version 2004). Safety and tolerability data were described by summary statistics and frequency tables.

\section{Results}

A total of 32 subjects participated in the study: eight healthy subjects, one subject with mild impairment of renal function, seven patients with moderate impairment of renal function, eight patients with severe impairment of renal function and eight patients with end-stage renal insufficiency requiring haemodialysis. More male subjects $(65.6 \%)$ were enrolled compared with female subjects (34.4\%). Table 1 summarizes the demographic characteristics of the study population. The groups compared well. However, healthy subjects were slightly younger and patients with moderate impairment of renal function older than the other groups. Concomitant diseases reported at study entry for subjects with chronic renal insufficiency were mostly related to their underlying disease. All subjects with impairment of renal function were taking concomitant medication at study entry and throughout the study, primarily for treatment of renal insufficiency and associated diseases. Two healthy subjects received concomitant medication (paracetamol for headache; ascorbic acid + calcium + rutoside for serous nose discharge; ascorbic acid + chlorhexidine for throat reddening) which was not considered to impact on the study objective. All 32 subjects completed the study and were included in the per protocol and safety analysis. Since only one subject with mild impairment of renal function enrolled in the study, an ANOVA was not possible for this group and this group could therefore not be included in comparative pharmacokinetics.

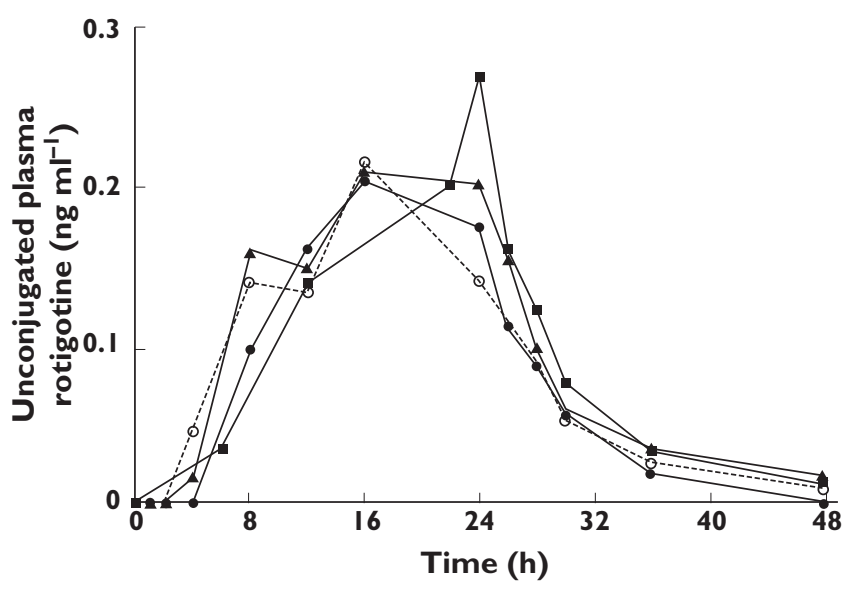

Figure 1

Median plasma concentration-time profiles of unconjugated rotigotine. $\bigcirc$ group 1 healthy subjects, $@$ group 3 moderate renal impairment, - group 4 severe renal impairment and $\boldsymbol{\square}$ group 5 end-stage renal impairment

\section{Pharmacokinetics}

The apparent dose values for rotigotine as an estimate for the amount of drug absorbed within $24 \mathrm{~h}$ were similar for all groups (ranging from a mean value of $1.95 \mathrm{mg}$ for patients with end-stage renal insufficiency to a mean value of $2.3 \mathrm{mg}$ for patients with severe impairment of renal function) with a mean value of $2.14 \mathrm{mg}$ which corresponds to $45.2 \%$ of the applied dose.

Unconjugated rotigotine Median plasma concentrationtime curves of unconjugated rotigotine are shown in Figure 1.

Curves were similar for all groups with maximum concentrations $16 \mathrm{~h}$ after patch application except for patients with end-stage renal insufficiency who showed maximum values at $24 \mathrm{~h}$. Blood sampling times were, however, different for these patients compared with the other groups due to haemodialysis procedures. Rotigotine was eliminated with a terminal half-life of approximately $7 \mathrm{~h}$. Most rotigotine concentrations had reached LOQ $24 \mathrm{~h}$ after patch 
removal in almost all subjects. Patients with end-stage renal insufficiency started dialysis $22 \mathrm{~h}$ after patch application. Their rotigotine elimination pattern was not different from the other groups indicating that rotigotine was not removed from plasma by haemodialysis. In accordance, no rotigotine was found in the dialysis fluid.

Table 2 presents the summary for the pharmacokinetic parameters for unconjugated rotigotine. Variability between subjects was high for most parameters. $C_{\max }$ increased slightly with the degree of renal insufficiency. The same trend was also observed for $A \cup C\left(0, t_{\text {last }}\right)$. Statistical comparison of the primary parameters $\left(C_{\max }\right.$ and $\left.\operatorname{AUC}\left(0, t_{\text {last }}\right)\right)$ for the different patient populations with renal impairment compared with healthy subjects showed that all point estimates for the ratios were close to 1:0.88 for AUC and 0.93 for $C_{\max }$ for moderate renal impairment, 1.14 and 1.18 for severe renal impairment and 1.05 and 1.25 for end-stage renal insufficiency requiring haemodialysis (Table S1 in supplementary file). No statistical correlation was observed between either $C_{\max }$ or $\operatorname{AUC}\left(0, t_{\text {last }}\right)$ for unconjugated rotigotine and creatinine clearance for healthy subjects and subjects with moderate and severe impairment of renal function $\left(\rho_{\mathrm{sp}}=-0.045, P=0.834\right.$ for $C_{\max }$ and $\rho_{\mathrm{sp}_{\mathrm{p}}}=-0.097, P=0.654$ for $\left.\mathrm{AUC}\left(0, t_{\text {last }}\right)\right)$. Median time to reach $C_{\max }$ was $16 \mathrm{~h}$ for healthy subjects and subjects with moderate impairment of renal function and $24 \mathrm{~h}$ for more severe renal insufficiency. There was no obvious difference in terminal half-life with mean values in the range of $5-7 \mathrm{~h}$ across the four treatment groups. Geometric mean values of $6.99 \mathrm{~h}$ and $6.8 \mathrm{~h}$ were observed for healthy subjects and end-stage renal insufficiency patients, respectively. The apparent total clearance and volume of distribution were similar across all four groups. On the other hand, Ae and renal clearance decreased with the degree of renal impair- ment. Statistical correlations with creatinine clearance were observed for Ae $\left(\rho_{s p}=0.433, P=0.039\right)$ and renal clearance $\left(\rho_{\mathrm{sp}}=0.416, P=0.048\right)$ but not for $\mathrm{CL} / F\left(\rho_{\mathrm{sp}}=\right.$ $0.085, P=0.692$ ). It should be noted, however, that renal clearance of unconjugated rotigotine accounts for only a very small percentage of the administered dose (Ae for healthy subjects was $0.02 \%$ of the administered rotigotine dose and ranged between $0.002 \%$ and $0.01 \%$ for subjects with renal impairment). Changes in renal clearance with increasing renal impairment had no observable effects on the apparent total clearance. Linear regression analysis established a correlation for $\mathrm{CL}_{R}$ and $\mathrm{CL}_{c r}\left(r^{2}=0.2864\right.$; supplementary Figure $\mathrm{S} 1 \mathrm{~A})$ but none for $\lambda_{z}$ with $\mathrm{CL}_{\mathrm{cr}}\left(r^{2}=\right.$ 0.0035; supplementary Figure S1B).

Protein binding could not be evaluated in some samples because rotigotine concentrations were below LOQ in one of the two dialysis chambers. Data above LOQ were available from nine subjects (mainly from the severe and end-stage groups). Median binding was similar in both groups ( $89 \%$ for patients with severe impairment of renal function and $91.9 \%$ for patients with end-stage renal insufficiency). Owing to sparse data, a correlation between the unbound fraction and serum albumin concentrations or $\mathrm{CL}_{\mathrm{cr}}$ could not be determined.

Conjugated rotigotine Median plasma concentrationtime curves of conjugated rotigotine are shown in Figure 2. Concentrations are about three-fold higher than for unconjugated rotigotine. Median conjugated rotigotine plasma concentration peaked $16 \mathrm{~h}$ after patch application for healthy subjects, $24 \mathrm{~h}$ for patients with moderate and with severe impairment of renal function, and $22 \mathrm{~h}$ for patients with end-stage renal insufficiency requiring dialysis (Figure 2). Plasma concentrations of conjugated

Table 2

Pharmacokinetic parameters of unconjugated rotigotine

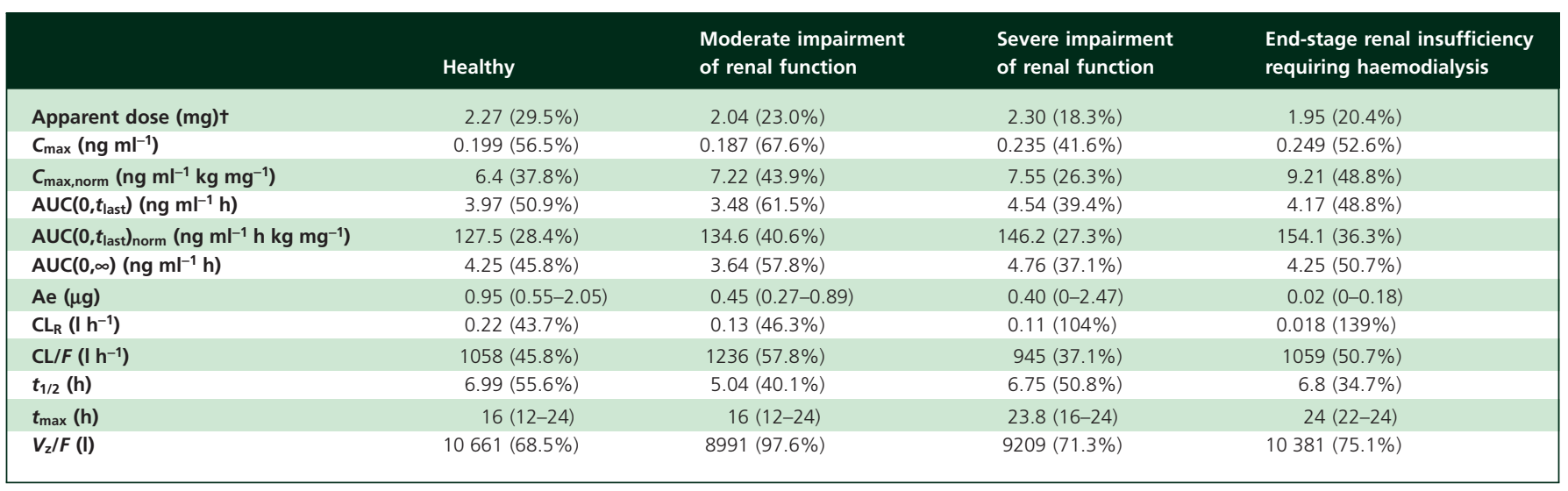

Data are expressed as geometric means (CV) or median (range). Norm, normalized to bodyweight and apparent dose, tarithmetic mean (CV). $C_{\text {max }}$ peak plasma concentration, $C_{\text {max, norm }}$ peak plasma concentration normalized by bodyweight and apparent dose, AUC $\left(0, t_{\text {last }}\right)$ area under the plasma concentration-time curve from time 0 to the last time point with a quantifiable concentration, $\mathrm{AUC}\left(0, \mathrm{t}_{\text {last }}\right)_{\text {norm }}$ area under the plasma concentration-time curve from time 0 to the last time point with a quantifiable concentration normalized by bodyweight and apparent dose, $\mathrm{AUC}(0, \infty)$ area under the plasma concentration-time curve from time 0 to infinity, Ae total amount excreted in urine, $\mathrm{CL}$ renal clearance, $\mathrm{CL} / \mathrm{F}$ total clearance of medication from plasma, $t_{1 / 2}$ terminal elimination half-life, $t_{\max }$ time to maximum plasma concentration, $\mathrm{V} z / \mathrm{F}$ fictive volume of distribution. 
rotigotine were considerably higher for patients with severe impairment of renal function; the peak of median conjugated rotigotine concentrations in this group (0.99 $\mathrm{ng} \mathrm{ml}^{-1}$ ) was more than double the concentration for healthy subjects $\left(0.35 \mathrm{ng} \mathrm{ml}^{-1}\right)$ and for patients with moderate impairment of renal function $\left(0.62 \mathrm{ng} \mathrm{ml}^{-1}\right)$. At the end of the dialysis comparison of median plasma concentrations of conjugated rotigotine measured in samples from the arterial inlet with corresponding concentrations in samples from the venous outlet reflected no extracorporeal extraction, $0.39 \mathrm{ng} \mathrm{ml}^{-1}$ vs. $0.43 \mathrm{ng} \mathrm{ml}^{-1}$.

Variability between subjects was high for most pharmacokinetic parameters (Table 3 ). For conjugated rotigotine, $C_{\max }$ and $\operatorname{AUC}\left(0, t_{\text {last }}\right)$ and their normalized values

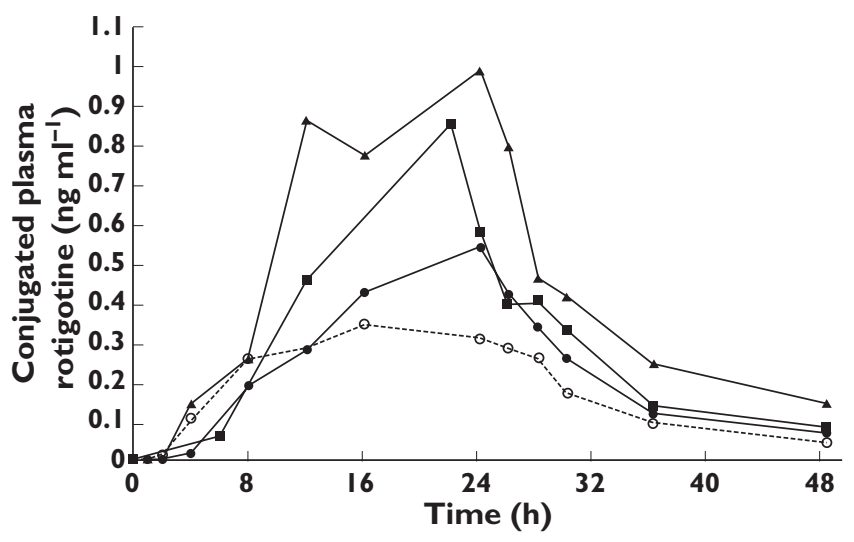

\section{Figure 2}

Median plasma concentration-time profiles of conjugated rotigotine. $\bigcirc$ group 1 healthy subjects, $\bigcirc$ group 3 moderate renal impairment, $\boldsymbol{\Delta}$ group 4 severe renal impairment and $\square$ group 5 end-stage renal impairment increased with decreasing renal function, particularly for the severe impairment group. The mean maximum plasma concentration $\left(C_{\max }\right)$ in this group was higher than for the group on dialysis. Statistical analysis indicated that the exposure to conjugated rotigotine was only slightly increased in subjects with moderate impairment of renal function compared with healthy subjects but $C_{\max }$ and $\operatorname{AUC}\left(0, t_{\text {last }}\right)$ doubled in subjects with severe impairment of renal function compared with healthy subjects (Table 3 ) with point estimates of 2.2 for $C_{\max }$ and 2.18 for AUC (supplementary Table S1). Intermediate increases in $C_{\max }$ and $A \cup C\left(0, t_{\text {last }}\right)$ of about $55 \%$ and $50 \%$ were observed in subjects with end-stage renal insufficiency requiring haemodialysis. However, it should be noted that the $90 \% \mathrm{Cls}$ were very wide and the variability in the data was high, particularly for the severe renal impairment group.

Correlations with $\mathrm{CL}_{\mathrm{cr}}$ were observed for both parameters: $\rho_{\mathrm{sp}}=-0.43, P=0.036$ for $C_{\max }$ and $\rho_{\mathrm{sp}}=-0.406, P=$ 0.049 for $A U C\left(0, t_{\text {last }}\right)$. Median time to reach $C_{\max }$ was identical for healthy subjects and subjects with different stages of renal insufficiency. Compared with healthy subjects, terminal half-life was slightly lower in subjects with moderate and end-stage renal insufficiency but higher in subjects with severe impairment of renal function. Hence, there was no obvious difference for terminal half-life with decreasing renal function. There was a general trend for decreasing apparent total clearance and volume of distribution with increasing severity of renal insufficiency; the lowest values were observed in the subjects with severe impairment of renal function. Ae and renal clearance were substantially lower in the groups with impaired renal function compared with healthy subjects: five times lower in the group with moderate impairment of renal function and four times lower for subjects with severe impairment of renal

\section{Table 3}

Pharmacokinetic parameters of conjugated rotigotine

\begin{tabular}{|c|c|c|c|c|}
\hline & Healthy & $\begin{array}{l}\text { Moderate renal } \\
\text { impairment }\end{array}$ & $\begin{array}{l}\text { Severe renal } \\
\text { impairment }\end{array}$ & $\begin{array}{l}\text { End-stage renal insufficiency } \\
\text { requiring haemodialysis }\end{array}$ \\
\hline$C_{\max }\left(\mathrm{ng} \mathrm{ml}^{-1}\right)$ & $0.492(46.5 \%)$ & $0.629(40.2 \%)$ & $1.08(99.1 \%)$ & $0.663(75.7 \%)$ \\
\hline $\operatorname{AUC}\left(0, t_{\text {last }}\right)\left(\mathrm{ng} \mathrm{ml}^{-1} \mathrm{~h}\right)$ & $10.8(48.0 \%)$ & $12.0(32.4 \%)$ & $23.5(90.0 \%)$ & $14.1(66.4 \%)$ \\
\hline $\operatorname{AUC}\left(0, t_{\text {last }}\right)_{\text {norm }}\left(\mathrm{ng} \mathrm{ml}^{-1} \cdot \mathrm{h} \cdot \mathrm{kg} \mathrm{mg}^{-1}\right)$ & $347.1(50.3 \%)$ & $475.1(33.4 \%)$ & $757.0(96.4 \%)$ & $520.9(44.2 \%)$ \\
\hline $\operatorname{AUC}(0, \infty)\left(\mathrm{ng} \mathrm{ml}^{-1} \mathrm{~h}\right)$ & $11.4(48.0 \%)$ & $12.9(31.7 \%)$ & $24.6(88.9 \%)$ & $14.7(64.7 \%)$ \\
\hline Ae $(\mu \mathrm{g})$ & $148.6(41.1-395.6)$ & $24.9(20.3-61.5)$ & $42.2(11.0-110.6)$ & $2.0(0.2-8.5)$ \\
\hline$t_{1 / 2}(h)$ & $10.2(33.2 \%)$ & $8.3(32.7 \%)$ & $11.1(10.4)$ & $9.4(15.5 \%)$ \\
\hline$t_{\max }(\mathrm{h})$ & $23.5(12-26)$ & $23.5(23.5-24)$ & $24(16-26)$ & $22(22-28)$ \\
\hline$\lambda_{z}\left(1 \mathrm{~h}^{-1}\right)$ & $0.068(33.2 \%)$ & $0.0836(32 . \%)$ & $0.0625(10.4 \%)$ & $0.0739(15.5 \%)$ \\
\hline
\end{tabular}

Data are expressed as geometric means (CV) or median (range). $C_{\text {max }}$ peak plasma concentration, $C_{\text {max,norm }}$ peak plasma concentration normalized by bodyweight and apparent dose, $A \cup C\left(0, t_{\text {last }}\right)$ area under the plasma concentration-time curve from time 0 to the last time point with a quantifiable concentration, $A \cup C\left(0, t_{\text {last }}\right)$ norm area under the plasma concentration-time curve from time 0 to the last time point with a quantifiable concentration normalized by bodyweight and apparent dose, $\mathrm{AUC}(0, \infty)$ area under the plasma concentration-time curve from time 0 to infinity, Ae total amount excreted in urine, $C L_{R}$ renal clearance, $C L / F$ total clearance of medication from plasma, $t_{1 / 2}$ terminal elimination half-life, $t_{\max }$ time to maximum plasma concentration, $\lambda_{z}$ terminal rate constant 
function. Renal clearance of conjugated rotigotine was almost negligible in subjects with end-stage renal insufficiency requiring haemodialysis. The correlation between conjugated rotigotine and creatinine clearance for $C_{R}$ and Ae was strong: $\rho_{S p}=0.863, P<0.0001$ for $C_{R}$, and $\rho_{S p}=$ $0.629, P=0.001$ for $A e$, but not for $C L / F: \rho_{s p}=0.376, P=$ 0.071 . Linear regression analysis established a correlation for $\mathrm{CL}_{R}$ and $\mathrm{CL}_{\mathrm{cr}}\left(r^{2}=0.7592\right.$; supplementary Figure $\left.\mathrm{S} 2 \mathrm{~A}\right)$ but none for $\lambda_{z}$ with $\mathrm{CL}_{c r} \quad\left(r^{2}=0.0112\right.$; supplementary Figure S2B).

$\mathrm{N}$-desalkyl metabolites Total N-desalkyl metabolites of rotigotine (unconjugated $\mathrm{N}$-desalkyl metabolites and conjugates) in plasma were determined $23.5 \mathrm{~h}$ after patch application ( $22 \mathrm{~h}$ for the end-stage group) and were higher in all groups with subjects with impaired renal function compared with healthy subjects (supplementary Table S2). Concentrations of unconjugated $\mathrm{N}$-despropyl and $\mathrm{N}$ desthienylethyl metabolites in plasma and urine were below LOQ for the majority of samples across all groups.

The amount excreted over $24 \mathrm{~h}$ for total $\mathrm{N}$-despropyl and $\mathrm{N}$-desthienylethyl metabolites varied between the groups (supplementary Table S2). There was no obvious trend for a decrease with increasing severity of renal insufficiency.

Safety

In total, 13 subjects reported 29 treatment emergent adverse events, most of which were mild or moderate in intensity. None of the AEs occurred in the group with endstage renal insufficiency. No serious AEs or deaths occurred during the study. Two severe AEs (headache and elevated blood pressure) were reported for a subject with moderate impairment of renal function. Another patient with moderate impairment of renal function had a mild upper respiratory tract infection which deteriorated into an acute bronchitis. The number of adverse events did not increase with the severity of renal insufficiency and all adverse events resolved by the end of the study. Seventy-two percent $(72 \%)$ of all AEs were considered related to the study medication including all events of nausea $(21.9 \%$, two healthy subjects, five subjects with impaired renal function), vomiting (9.4\%, one healthy subject, two subjects with impaired renal function) and application site reactions $(9.4 \%$, two healthy subjects, one subject with impaired renal function). The latter were described as 'itching under the patch' and were mild in intensity. Evaluation of skin tolerability revealed one case of evident and eight cases of slight erythema after $24 \mathrm{~h}$ of patch application as well as one slight and two marginal oedemas. Overall patch adhesiveness was good. The majority of patients did not show any patch lift (72\%) and only four subjects presented with a $20 \%-49 \%$ lift.

There were no clinically relevant changes in vital signs, physical examination or ECG findings during the study. Laboratory values differed between the groups consistent with the underlying condition of the subjects with impaired renal function compared with healthy subjects but there were no clinically relevant changes in any group.

\section{Discussion}

Exposure to the active substance, unconjugated rotigotine, was similar in healthy subjects and subjects with different stages of renal insufficiency as indicated by similar plasma concentration-time curves and pharmacokinetic parameters $\operatorname{AUC}\left(0, t_{\text {last }}\right)$ and $C_{\text {max. }}$. Bioavailability of unconjugated rotigotine was not affected by renal insufficiency. Point estimates for the ratio between healthy subjects and renal impaired subjects for $A \cup C\left(0, t_{\text {last }}\right)$ and $C_{\max }$ were near 1 . The respective $90 \%$ Cls were quite wide owing to the low subject number in each group. The lack of a correlation of these parameters with $\mathrm{CL}_{\mathrm{cr}}$ supports the findings for unconjugated rotigotine. The terminal half-life of rotigotine was also comparable between the groups. Renal elimination of unconjugated rotigotine decreased with increasing severity of renal insufficiency but had no relevant effect on the total clearance. This can be explained by the extremely low percentage of unconjugated rotigotine $(<1 \%$ of the administered dose) excreted in urine. Plasma concentrations did not decrease in the central circulation under extracorporeal haemodialysis, indicating that the compound was not extracted by haemodialysis.

Median plasma protein binding data measured in this study are in accordance with the plasma protein binding assessed in vitro (91.6\%). The fact that rotigotine is not extracted from plasma by haemodialysis is mainly due to its high protein binding.

Conjugated rotigotine concentrations increased with impairment of renal function but there was no obvious trend for terminal half-life. Statistical comparison with healthy subjects revealed point estimates for the ratio of $\operatorname{AUC}\left(0, t_{2}\right)$ and $C_{\max }$ of approximately 1.4 in subjects with moderate impairment of renal function, approximately 1.5 in subjects with end-stage renal insufficiency and 2.2 in subjects with severe impairment of renal function. Both parameters correlated with $\mathrm{CL}_{\mathrm{cr}}$. Whereas the renal clearance of conjugated rotigotine showed a correlation with the extent of renal function, the rate constant of elimination showed no influence of renal function on the rate of elimination as a whole. This indicates that biliary excretion is relevant as alternative elimination route for conjugated rotigotine.

The pharmacological activity of rotigotine and its metabolites has been tested in in vitro receptor binding assays with human receptor subtypes expressed in cell lines. Whereas rotigotine exhibits its high affinity for the dopamine receptors, in particular for the $D_{3}$ receptor, the phase 2 conjugates have practically no affinity (data on file). Therefore, the increase in exposure to conjugated rotigotine observed in subjects with impaired renal 
function (up to approximately two-fold in subjects with severe impairment of renal function) is considered not relevant for biological activity of rotigotine.

In vitro, unconjugated N-desalkyl metabolites also have a high affinity to some of the dopamine receptors. However, unconjugated N-desalkyl metabolites are hardly detectable in plasma in healthy subjects as well as in subjects with different stages of renal insufficiency. Plasma concentrations of total $\mathrm{N}$-desalkyl metabolites are increased in subjects with impaired renal function compared with healthy subjects. This reflects mainly an increase in conjugates of $\mathrm{N}$-desalkyl metabolites which are also considered biologically inactive.

Taking into consideration all the pharmacokinetic assessments in this study, dose adjustment is not needed for rotigotine treatment in patients with different stages of renal insufficiency including patients requiring haemodialysis.

End-stage renal disease is often associated with RLS. In a recent cross-sectional study of ESRD patients requiring haemodialysis, one fifth of all patients $(21.5 \%)$ were affected by RLS, with negative impact on quality of sleep, including higher risk of sleep apnoea and more depressive symptoms than in patients without RLS [20]. The incidence of new cardiovascular events was also higher in ESRD patients with RLS, with a significantly higher risk of mortality [15].

Overall prevalence of PD increases with age [13]. Also, impairment of renal function increases with age [16], having important implications for drug therapy in the elderly [17]. Hence, the availability of a dopaminergic treatment option without the need for dose adjustment due to a decrease in renal function may be an advantage in patients suffering from PD or RLS.

The influence of renal insufficiency on the pharmacokinetic profile of other dopamine agonists administered for PD and RLS treatment has been investigated. Oral pramipexole is almost completely eliminated by renal tubular secretion [21] and therefore dose adjustments are needed for patients with moderate to severe impairment of renal function. Oral clearance values of ropinirole were not different in patients with moderate impairment of renal function compared with patients with mild or no impairment of renal function [22]. The effect of haemodialysis on ropinirole removal is not known but considered unlikely because of the relatively high apparent volume of distribution of this dopamine agonist [23]. Data for patients with severe renal insufficiency are not available. The pharmacokinetics of cabergoline were not altered in 12 patients with moderate-to-severe renal insufficiency [24]. The probable reason for this finding is the same as for rotigotine: a very low renal elimination of $1 \%$ for the active compound [25].

Application of the rotigotine patch was well tolerated in both healthy subjects and subjects with impaired renal function. The most frequently reported adverse events were nausea and vomiting, common dopaminergic side effects which were mild to moderate in intensity and resolved by the end of the study. Both skin tolerability and adhesiveness of the transdermal rotigotine patch were good.

In conclusion, no dose adjustment for transdermal rotigotine is required in subjects with different stages of impaired renal function including subjects with end-stage renal insufficiency requiring haemodialysis. This may be of advantage in the chronic treatment of an older patient population, e.g. patients with PD or RLS.

\section{Competing Interests}

The study was sponsored by Schwarz Biosciences, a member of the UCB Group of companies, Germany. All costs associated with the development and the publishing of the present manuscript were met by the sponsor. WC, $S A$ and $M B$ are employees of the study sponsor. WS and ASM were investigators contracted to carry out the clinical part of the study.

AAl Deutschland GmbH\&Co.KG, NeuUlm, Germany performed all bioanalytical work to quantify rotigotine and metabolites in plasma and urine samples. In addition, $A A I$ determined protein binding in selected plasma samples. The authors are grateful to E. Grosselindemann (Brett Medical Writing Australia) for writing assistance and to G.-G. Li (UCB Pharma SA) for publication coordination.

\section{REFERENCES}

1 Pfeiffer RF. A promising new technology for Parkinson's disease. Neurology 2005; 65: (Suppl. 1): S6-10.

2 Chase TN. The significance of continuous dopaminergic stimulation in the treatment of Parkinson's disease. Drugs 1998; 55: (Suppl. 1): 1-9.

3 Cawello W, Wolff HM, Meuling WJA, Horstmann R, Braun M. Transdermal administration of radiolabelled [14C]-rotigotine by a patch formulation. A mass balance trial. Clin Pharmacokinet 2007; 46: 851-7.

4 Cawello W, Braun M, Boekens H. Absorption, disposition, metabolic fate, and elimination of the dopamine agonist rotigotine in man - administration by intravenous infusion or transdermal delivery. Drug Metab Dispos 2009; 37: 2055-60.

5 Braun M, Cawello W, Andreas J-O, Boekens H, Horstmann R. Lack of pharmacokinetic interactions between transdermal rotigotine and oral levodopa/carbidopa. J Clin Pharmacol 2009; 49: 1047-55.

6 Braun M, Cawello W, Boekens H, Horstmann R. Influence of domperidone on pharmacokinetics, safety and tolerability of the dopamine agonist rotigotine. Br J Clin Pharmacol 2009; 67: 209-15. 
7 The Parkinson Study Group. A controlled trial of rotigotine monotherapy in early Parkinson's disease. Arch Neurol 2003; 60: 1721-8.

8 Watts RL, Jankovic J, Waters C, Rajput A, Boroojerdi B, Rao J. Randomized, blind, controlled trial of transdermal rotigotine in early Parkinson disease. Neurology 2007; 68: 272-6.

9 LeWitt PA, Lyons KE, Pahwa R. Advanced Parkinson disease treated with rotigotine transdermal system (PREFER Study). Neurology 2007; 68: 1262-7.

10 Poewe WH, Rascol O, Quinn N, Tolosa E, Oertel WH, Martignoni E, Rupp M, Boroojerdi B. Efficacy of pramipexole and transdermal rotigotine in advanced Parkinson's disease: a double-blind, double-dummy, randomised controlled trial. Lancet Neurol 2007; 6: 513-20.

11 Oertel WH, Beneš H, Garcia-Borreguero D, Geisler P, Högl B, Saletu B, Trenkwalder C, Sommerville KW, Schollmayer E, Kohnen R, Stiasny-Kolster K. Efficacy of rotigotine transdermal system in severe restless legs syndrome: a randomized, double-blind, placebo-controlled, six-week dose-finding trial in Europe. Sleep Med 2008; 9: 228-39.

12 Trenkwalder C, Beneš H, Poewe W, Oertel WH, García-Borreguero D, de Weerd AW, Ferini-Strambi L, Montagna P, Odin P, Stiasny-Kolster K, Högl B, Chaudhuri KR, Partinen M, Schollmayer E, Kohnen R. Efficacy of rotigotine for treatment of moderate-to-severe restless legs syndrome: a randomised, double-blind, placebo-controlled trial. Lancet Neurol 2008; 7: 595-604.

13 de Rijk MC, Launer LJ, Berger K, Breteler MM, Dartigues JF, Baldereschi M, Fratiglioni L, Lobo A, Martinez-Lage J, Trenkwalder C, Hofman A. Prevalence of Parkinson's disease in Europe: a collaborative study of population-based cohorts. Neurology 2000; 54: (Suppl. 5): S21-3.

14 García-Borreguero D, Egatz R, Winkelmann J, Berger K. Epidemiology of restless legs syndrome: the current status. Sleep Med Rev 2006; 10: 153-67.

15 La Manna G, Pizza F, Persici E, Baraldi O, Comai G, Cappuccilli ML, Centofanti F, Carretta E, Plazzi G, Coli L, Montagna P, Stefoni S. Restless legs syndrome enhances cardiovascular risk and mortality in patients with end-stage kidney disease undergoing long-term haemodialysis treatment. Nephrol Dial Transplant 2010; (Epub ahead of print).

16 Beck LH. Aging changes in renal function. In: Principles of Geriatric Medicine and Gerontology, $4^{\text {th }}$ edn. eds Hazzard WR, Blass JP, Ettinger WH, Halter JB, Ouslander JG. New York: McGraw-Hill, 1999; 767-76.

17 Epstein M. Aging and the kidney. J Am Soc Nephrol 1996; 7: 1106-22.

18 U.S. Department of Health and Human Services, Food and Drug Administration guidance for industry. Pharmacokinetics in patients with impaired renal function study design, data analysis, and impact on dosing and labelling. May 1998.
19 EMEA Guidance on the evaluation of the pharmacokinetics of medical products in patients with impaired renal function, CHMP/EWP/225/02, June 2004.

20 Araujo SM, de Bruin VM, Nepomuceno LA, Maximo ML, Daher EF, Correia Ferrer DP, de Bruin PF. Restless legs syndrome in end-stage renal disease: clinical characteristics and associated comorbidities. Sleep Med 2010; 11: 785-90.

21 Bennett JP Jr, Piercey MF. Pramipexole: a new dopamine agonist for the treatment of Parkinson's disease. J Neurol Sci 1999; 163: 25-31.

22 Kaye CM, Nicholls B. Clinical pharmacokinetics of ropinirole. Clin Pharmacokinet 2000; 39: 243-54.

23 Requip $^{\circledR}$ (ropinirole tablets): GlaxoSmithKline prescribing information. Available at http://us.gsk.com/products/ assets/us_requip.pdf (last accessed 22 Jul 2011).

24 Del Dotto P, Bonuccelli U. Clinical pharmacokinetics of cabergoline. Clin Pharmacokinet 2003; 42: 633-45.

25 Deleu D, Northway MG, Hanssens Y. Clinical pharmacokinetic and pharmacodynamic properties of drugs used in the treatment of Parkinson's disease. Clin Pharmacokinet 2002; 41:261-309.

\section{Supporting Information}

Additional Supporting Information may be found in the online version of this article:

Figure S1 (A) Correlation of renal clearance of unconjugated rotigotine with creatinine clearance; $(\boldsymbol{O})$ individual estimations, (-) result of linear regression. (B) Correlation of rate constant of unconjugated rotigotine elimination with creatinine clearance; $(0)$ individual estimations, (-) result of linear regression

Figure S2 (A) Correlation of renal clearance of conjugated rotigotine with creatinine clearance; $(\mathbf{O})$ individual estimations, (-) result of linear regression. (B) Correlation of rate constant of conjugated rotigotine elimination with creatinine clearance; $(0)$ individual estimations, $(-)$ result of linear regression

Table S1 Statistical comparison of $C_{\max }$ and $\operatorname{AUC}\left(0, t_{\text {last }}\right)$ between patient groups with different stages of renal insufficiency and healthy subjects (point estimates [least squares means] and $90 \%$ confidence interval)

Table S2 Pharmacokinetic parameters of total N-desalkyl metabolites of rotigotine

Please note: Wiley-Blackwell are not responsible for the content or functionality of any supporting materials supplied by the authors. Any queries (other than missing material) should be directed to the corresponding author for the article. 